\title{
Contribution à l'étude du cycle évolutif d'un Cestode du genre Proteocepbalus parasite de Coregonus fera, en provenance du Lac Léman
}

\author{
Par Léna JARECKA et J.-M. DOBY
}

En août 1963, il nous fut possible de trouver en abondance, dans les intestins de plusieurs Coregonus fera en provenance de divers points du Lac Léman, un Protéocéphale. En effet, certains des poissons hébergeaient de 20 à 30 parasites. Ces Cestodes étaient alors tous à maturité et présentaient un utérus rempli d'œufs.

Grâce à cet abondant matériel, nous avons pu tenter de réaliser le cycle expérimental de ce Protéocéphale, observant du moins le développement de l'embryon dans l'hôte intermédiaire jusqu'à la totale maturité de la larve.

\section{A) Obtention des œufs.}

Ces Cestodes adultes vivants, placés dans de l'eau ordinaire, émettent spontanément leurs œufs ; il semble à ce sujet que l'utérus se vide par un point précis, et non par éclatement des parois et de la cuticule du parasite. Un orifice de «ponte » a d'ailleurs été décrit par plusieurs auteurs chez plusieurs espèces de Proteocephalus (La Rue, 1909 ; Cooper, 1921 ; Mac Lulich, 1943, etc...). Le Cestode se libère ainsi totalement et spontanément de ses œufs. Ceux-ci peuvent être à différents stades de maturité. Nous considérons comme œuf «mûr» un œuf qui présente ses trois enveloppes bien développées, son embryon avec crochets et qui, émis dans l'eau, garde intacte sa structure pendant plusieurs jours. Quand l'œuf n'est pas à maturité, sa structure dans l'eau se modifie rapidement après l'émission et l'onchosphère meurt.

\section{B) Infestation de Copépodes.}

Les œufs obtenus de la façon précitée furent placés dans de l'eau directement avec plusieurs espèces de Copépodes et de Cladocères d'origines diverses, et laissés en contact avec ces derniers pendant plusieurs jours. 
Voici les espèces de Crustacés qui furent utilisées pour l'expérimentation :

$1^{\circ}$ Crustacés en provenance de divers points du Lac Léman: Cyclops abyssorum (G.O. Sars 1863) (1) ; Eudiaptomus sp. (2) ; Leptodora kindtii (Focke 1844) ; Bythotrephes longimanus (Leydig).

$2^{\circ}$ Crustacés en provenance de bassins de la station de Pisciculture des eaux et FORÊTS DE THONON : Cyclops strenuus strenuus (Fischer 1851) (1).

$3^{\circ}$ Crustacés obtenus par élevage au laboratoire de Rennes, de provenances diverses de l'Ouest de la France: Macrocyclops albidus (Jurine 1820); Macrocyclops fuscus (Jurine 1820); Acanthocyclops gigas (Claus 1857); Acanthocyclops viridis (Jurine 1820) ; Eucyclops serrulatus (Fischer 1851).

Les tentatives d'infections expérimentales de ces divers Crustacés ont donné les résultats suivants (température de 17 à $20^{\circ}$ ) :

$1^{\circ}$ Chez Eudiaptomus sp., Leptodora kindtii, Bythotrephes longimanus, Macrocyclops albidus, Macrocyclops fuscus, Acanthocyclops gigas et Acanthocyclops viridis, il n'y eut aucune évolution, aussi minime soit-elle. En effet, aucun embryon ne fut trouvé dans la cavité générale.

$2^{\circ}$ Chez Eucyclops serrulatus, il y eut un début d'évolution. En effet, de nombreux embryons passèrent dans la cavité générale et y persistèrent vivants pendant vingt jours au moins, mais sans évoluer.

$3^{\circ}$ Enfin, chez Cyclops abyssorum et chez Cyclops strenuus strenuus, le développement fut complet :

\section{a) Chez Cyclops strenuus strenuus :}

- Vingt-quatre heures après le début du contact, les onchosphères bien mobiles se retrouvent dans la cavité générale du Copépode, et mesurent alors environ $30 \mu$.

- Au troisième jour qui suit l'infestation, on voit apparaître à l'intérieur de l'embryon un massif cellulaire nettement différencié, dans la partie qui est postérieure aux crochets. Cet embryon mesure alors de 40 à $50 \mu$.

- Le quatrième jour, le développement du massif cellulaire interne se poursuit et l'embryon mesure alors de 65 à $70 \mu$.

(1) Nous remercions vivement M. le Professeur F. Kiefer, du Anstalt für Bodenseeforschung der Stadt Konstanz, qui a bien voulu examiner notre matériel. Voici ses conclusion (in litt.): «Cyclops abyssorum G.O. Sars. Il s'agit d'une forme locale particulière, qui, à mon avis, n'a pas encore été étudiée jusqu'à présent d'une façon détaillée...

« Cyclops strenuus strenuus Fischer. Le matériel du bassin de la Pisciculture de Thonon est à rattacher, au mieux, à cette espèce. Cette détermination n'est cependant pas pleinement certaine, car, au cours de ces dernières années, il est apparu de plus en plus que, en raison de la proche parenté des différentes espèces du genre Cyclops, une série annuelle d'échantillons est pour le moins nécessaire à la détermination en certitude d'une population. Car les animaux de ce groupe de Cyclopidés sont en partie fortement variables selon la saison... »

(2) Ce Copépode fut déterminé Eudiaptomus vulgaris (Schmeil, 1896) par M. P. Laurent, que nous tenons à remercier également ici pour l'accueil si aimable qu'il nous a accordé dans la Station dont il a la responsabilité. Pour l'un de nous (Jarecka), il s'agirait peut-être plutôt de Eudiaptomus gracilö̈des (Lilljeborg, 1888). 
- Au cinquième jour, l'embryon commence à s'allonger, il mesure alors environ $65 \mu$ de large sur $90 \mu$ de long.

- Au neuvième jour, l'embryon mesure de 300 à $400 \mu$ dans son ensemble. Sa largeur est de $120 \mu$ environ au niveau du scolex et de $80 \mu$ dans la partie médiane. Il n'y a pas encore de ventouses ni de système excréteur visibles. La cuticule n'est pas encore différenciée, ni finement épineuse; cependant, un étranglement marque déjà l'apparition du cercomère.

Les crochets sont retrouvés alors répartis sans ordre particulier, principalement dans la partie médiane du corps, et non dans le cercomère. Des granulations calcaires commencent à apparaître ( 1 à $3 \mu$ de diamètre).

Ces derniers chiffres correspondent aux dimensions relevées sur des larves libérées par dilacération de l'hôte à frais, et non comprimées. Ils concernent des larves qui se trouvaient au nombre de trois dans un même exemplaire de Cyclops. Cependant, chez certains Cyclops infectés pourtant dans les mêmes délais, le développement de la larve s'est révélé plus lent; ainsi, par exemple, trois larves contenues dans un même Cyclops mesuraient respectivement 60 sur $50 \mu, 110$ sur $60 \mu, 180$ sur $60 \mu$; chez ces larves, aucun cercomère n'était encore différencié.

- Au bout de douze jours, la larve présente des ventouses déjà apparentes, mais encore incomplètement formées. La ventouse apicale se manifeste déjà par un petit massif cellulaire. La cuticule est alors bien différenciée et présente une spinulation fine, visible surtout en contraste de phase. Les épines ont environ $1 \mu$. Le système excréteur est visible, notamment les canaux principaux et la vésicule excrétrice. Les granulations calcaires sont plus nombreuses et plus volumineuses, atteignant un diamètre de 6 à $7 \mu$. Les crochets sont toujours visibles et répartis sans ordre dans les tissus. Le cercomère est alors très bien différencié et facilement détachable du reste du corps par simple manipulation.

Voici les dimensions relevées sur l'une de trois larves renfermées dans un même Cyclops :

— Longueur sans le cercomère : $350 \mu$.

- Cercomère : $90 \mu$ de long, plus renflé à l'extrémité, et d'une largeur médiane de $20 \mu$.

- Largeur au niveau du scolex : $100 \mu$ de large. Les ventouses, sur l'exemplaire légèrement comprimé entre lame et lamelle, mesuraient de 35 à $40 \mu$ de diamètre.

Le massif cellulaire de la cinquième ventouse mesurait $30 \mu$ de diamètre.

Les deux autres larves mesuraient respectivement $310 \mu$ sur $140 \mu$ et $340 \mu$ sur $130 \mu$.

- Au quatorzième jour après l'infestation, il semble que la plus grande partie de l'évolution soit terminée. En effet, voici les dimensions de deux larves au même stade trouvées dans un seul Cyclops :

$300 \mu$ sur $140 \mu$ (chiffres obtenus par mensuration dans le Cyclops non éclaté, ni comprimé). Ces deux larves ne présentaient plus de cercomères visibles. Les larves, extraites de la cavité du Cyclops, se contractent et s'invaginent dans l'eau; elles mesu- 
rent alors $180 \mu$ sur $160 \mu$. A certains moments, en extension longitudinale maximale, ces larves ont atteint $600 \mu$ de long sur $100 \mu$ de large au niveau du scolex.

Le système excréteur est alors bien visible (canaux, vésicule et cellules-flammes). La cuticule est très nettement spinulée sur environ les deux tiers antérieurs du corps. A l'intérieur des tissus, dans la zone post-ventousaire, apparaît une zone de cellules nettement plus granuleuses. Les crochets sont toujours visibles, répartis sans ordre, mais presque toujours dans la zone médiane du corps. Il existe de nombreuses granulations calcaires pouvant atteindre un diamètre de $10 \mu$. Elles peuvent être isolées, rondes, ovales, ou en amas d'éléments beaucoup plus petits, ou en chapelets de trois ou quatre, de taille moyenne.

En résumé, il semble que, quinze jours après le début de l'infestation, du moins dans les conditions de notre expérimentation, la larve ait atteint sa maturité. En effet, par la suite, il n'y a plus de modifications sensibles de la morphologie, sauf peut-être une petite augmentation de taille. La survie de ces larves dans la cavité de Cyclops strenuus peut être très longue puisque, soixante-dix jours après le début de l'infestation, des larves y ont été retrouvées encore vivantes. Voici la description d'une larve de cet âge :

- $550 \mu$ sur $130 \mu$;

— ventouses : diamètre de $50 \mu$;

— ventouse apicale : diamètre de 25 à $30 \mu$;

- les crochets sont toujours visibles.

Le cercomère reste également vivant, mais le plus souvent, il est détaché du reste du corps et se retrouve dans la cavité générale, bien mobile, parfois fort loin de la larve elle-même.

Dans les conditions de notre expérimentation, les taux d'infestation de Cyclops strenuus strenuus ont atteint $100 \%$, chaque Copépode hébergeant de une à sept larves (en moyenne trois). Nous avons dit plus haut que la larve restait vivante pendant longtemps dans la cavité générale du Cyclops. Cependant, si l'infestation concerne un Copépode non à maturité, les larves meurent dans la cavité générale au moment de la mue. C'est ainsi que, dans un élevage, on a pu observer une diminution progressive du taux d'infestation en raison de nombreuses mues. Par contre, dans un autre élevage, où tous les individus étaient arrivés à maturité avant l'infestation, le taux de celle-ci s'est maintenu.

Au cours de la dégénérescence, qui se manifeste après la mort de la larve dans la cavité générale du Copépode, on observe une vacuolisation des cellules et une disparition progressive des ventouses. Les granulations calcaires se dissolvent peu à peu, mais les crochets restent bien visibles jusqu'à la disparition totale.

\section{b) Chez Cyclops abyssorum :}

L'infestation a eu lieu dans les mêmes conditions que pour Cyclops strenuus. Cependant, il n'y a pas eu d'observation aussi suivie de l'évolution larvaire. Il semble 
toutefois que l'évolution chez Cyclops abyssorum soit comparable à celle qui prend place chez Cyclops strenuus; ainsi, trente jours après le début de l'infestation, la larve est déjà bien formée. Elle mesure $170 \mu$ de large sur $270 \mu$ de long. Les ventouses principales mesurent $50 \mu$ de diamètre, la ventouse apicale environ $30 \mu$. Ici aussi, sont bien visibles la spinulation cuticulaire, les crochets, le système excréteur, les granulations calcaires, la zone des cellules granuleuses.

Comme précédemment, nous avons pu observer une longue survie des larves dans la cavité générale des Cyclops, puisque les Copépodes étaient encore parasités 75 jours après l'infestation.

Malheureusement, la colonie de ces Cyclops pélagiques, en provenance du Léman, particulièrement difficile à maintenir en survie, mourut 84 jours après le début de la mise en expérimentation. Ici aussi, nous avons pu observer dans la cavité générale la survie du cercomère (piriforme, de 120 sur $60 \mu$ ). Cependant, chez Cyclops abyssorum, contrairement à ce que nous avons observé chez Cyclops strenuus, autant que nous avons pu en juger, le cercomère a été relativement peu souvent retrouvé.

Comme dans l'autre expérience, le taux d'infestation a été très élevé, presque à $100 \%$. Chaque Cyclops parasité hébergeait de une à six larves (moyenne trois).

COMPARAISON AVEC LE MATÉRIEL RÉCOLTÉ DIRECTEMENT DANS LA NATURE.

Les larves obtenues au cours des infestations expérimentales se montrèrent très peu différentes, sinon identiques, à une seule larve trouvée dans la nature chez Cyclops abyssorum en provenance du Léman, le 20 juillet. Cette larve, contractée et invaginée, mesurait $300 \mu$ sur $220 \mu$. Ses ventouses principales avaient un diamètre de $55 \mu$, la ventouse apicale $30 \mu$.

La spinulation cuticulaire était bien visible au contraste de phase, notamment sur les deux tiers antérieurs du corps. Les granulations calcaires les plus grosses atteignaient de 10 à $12 \mu$ et présentaient le même aspect que dans les larves obtenues expérimentalement. Le système excréteur s'est révélé également identique, notamment dans la trame du réseau des canaux dans la région péri-et post-ventousaire.

\section{Pourcentage D'infestation NATURelle.}

Deux prélèvements de zooplancton furent effectués dans le Léman, à deux endroits éloignés de plusieurs kilomètres: Cyclops abyssorum : un parasité par une larve sur 150 examinés. Aucun parasitisme chez 300 Eudiaptomus sp. (graciloides?), 150 Leptodora kindtii et 100 Bythotrephes longimanus.

\section{C) Essais d'infestation de Poissons à l'aide des Copépodes parasités.}

$1^{\circ}$ Cinq alevins de Cyprinidés sp., de $3 \mathrm{~cm}$ environ, chaque individu étant isolé, reçurent chacun cinq Cyclops abyssorum dont le parasitisme avait été vérifié, soit environ quinze larves de Protéocéphales, soixante jours après l'infestation des Copépodes. La surveillance des alevins et des Copépodes mis en contact nous permet d'affirmer 
l'ingestion des seconds par les premiers. Les alevins furent autopsiés dans des délais variables :

- Deux alevins furent sacrifiés douze heures après le repas infectant. Plusieurs larves vivantes furent retrouvées dans leur intestin.

- Un alevin fut sacrifié trente heures après le repas infectant; plusieurs larves vivantes furent encore retrouvées dans l'intestin.

- Enfin, deux alevins furent sacrifiés quarante-huit heures après le repas infectant. Aucune larve de Protéocéphale ne fut retrouvée dans leur intestin.

Cependant, plusieurs larves vivantes furent retrouvées dans les matières fécales des Poissons au fond des récipients. Il semble qu'il n'y ait donc pas eu de digestion des larves par le Poisson, mais plutôt que celles-ci aient effectué un simple transit sans fixation au niveau de la muqueuse et aient été rejetées dans le milieu extérieur.

FIG. 1. - Eufs avec leur membrane externe encore plissée, au moment de l'émission spontanée dans l'eau

FIG. 2. - Eufs dont la membrane externe a commencé à se déplisser, après de 5 à 10 minutes de séjour dans l'eau

FIG. 3. - Eufs dont la membrane atteint sa turgescence maximale, après de 20 à 30 minutes de séjour dans l'eau. Dans l'un de ces œufs, à la suite d'une légère pression, l'onchosphère, avec sa membrane, est sortie de la membrane interne éclatée

FIG. 4. - Onchosphère avec ses 3 membranes, dont l'externe est encore plissée

FIG. 5, - Détail d'un œuf avec éclosion de l'onchosphère

FIG. 6. - Crochets de l'embryon

FIG. 7. - Début de développement de l'embryon dans la cavité générale de Cyclops strenuus, trois jours après l'infestation de celui-ci

FIG. 8. - Idem., quatre jours après l'infestation.

FIG. 9. - Idem., cinq jours après l'infestation

FIG. 10. - Idem., neuf jours après l'infestation. Le cercomère commence à s'individualiser

FIG. 11. - Idem., douze jours après l'infestation. Le cercomère est bien individualisé. Les ventouses commencent à se former

FIG. 12. - Larve à maturité, âgée de quatorze jours, avec ventouses, système excréteur, granulations calcaires, et cercomère détaché

FIG. 13. - Larve de même âge, mais contractée et invaginée hors de la cavité générale du copépode

FIG. 14. - Détail de la partie antérieure de la larve dessinée en 12, montrant la cuticule finement spinulée, les canaux excréteurs, les granulations, la zone post-ventousaire des cellules granuleuses, un crochet de l'embryon inclus latéralement dans le parenchyme

FIG. 15. - Cercomère de la larve dessinée en 14, détaché de celle-ci et trouvé, bien mobile, à l'état libre, dans la cavité générale du Copépode

N. B. - Les lettres accompagnant chaque numéro de dessin renvoient à l'échelle micrométrique correspondante, dont une division $=10 \mathrm{u}$.

Certains de ces dessins présentent des irrégularités. La raison en est qu'ils ont été effectués à la chambre claire et n'ont été l'objet d'aucune schématisation. 


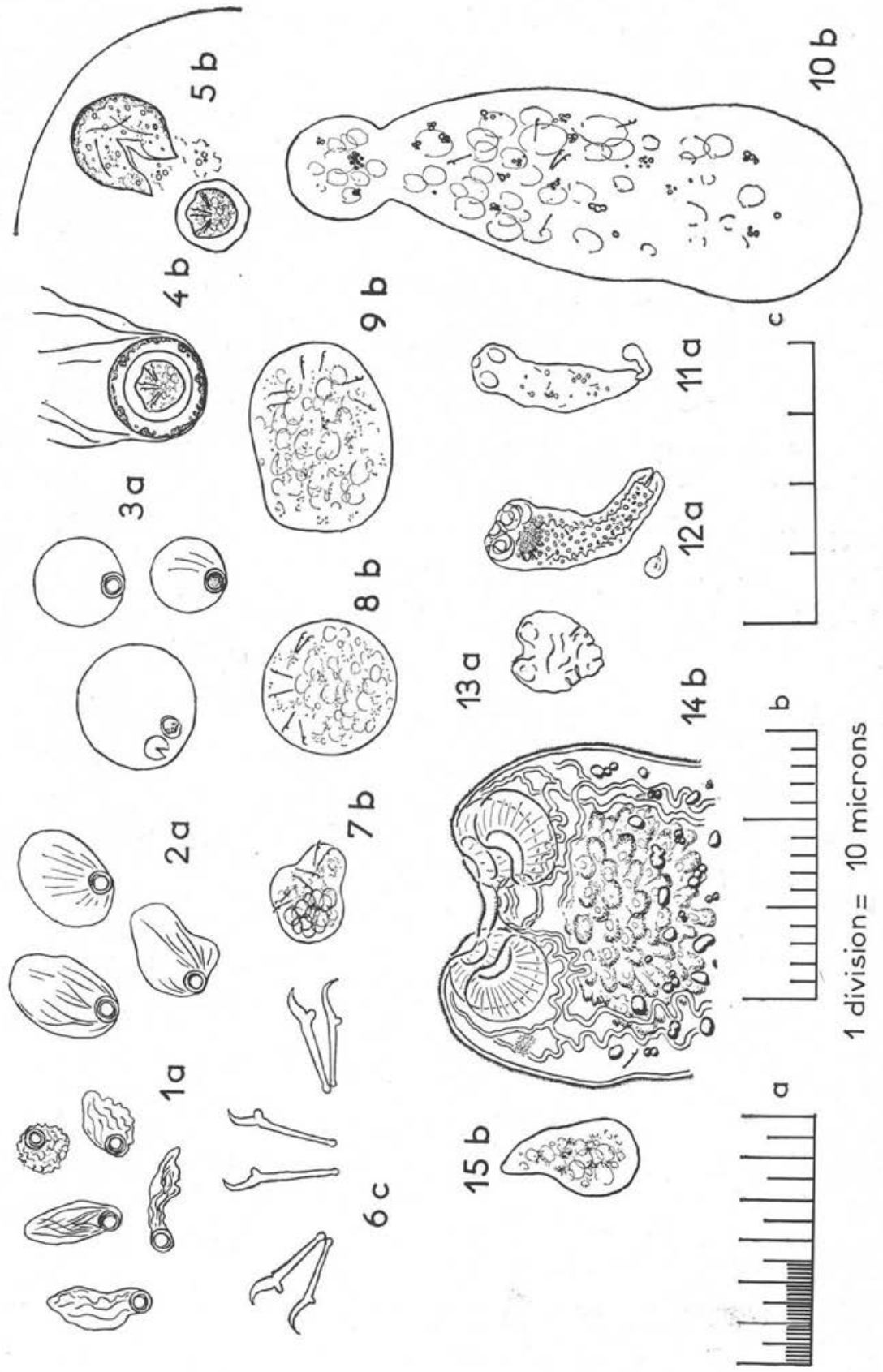


N
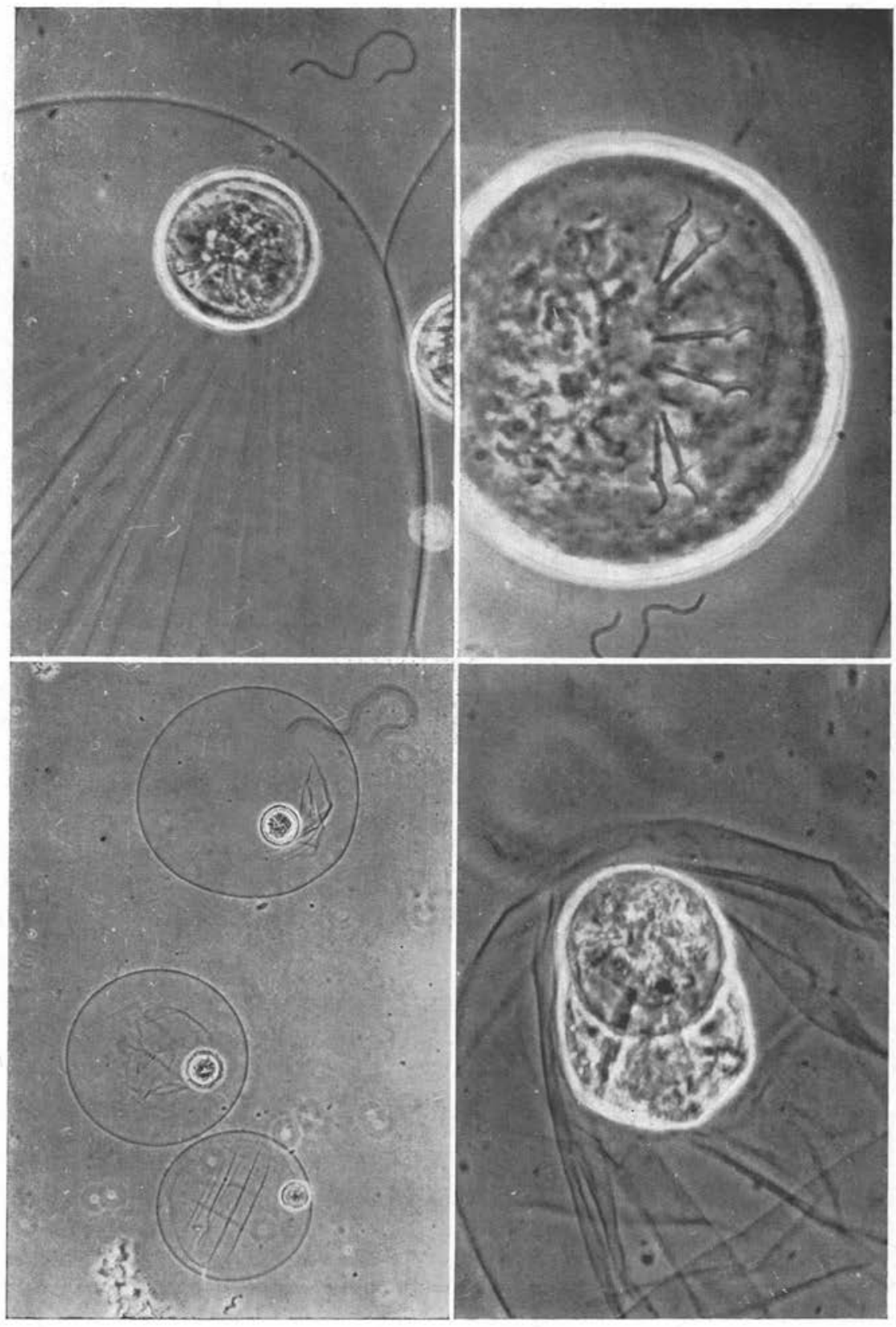

氙

윤

흔

등 ह

ป

$1=$

ก.

。

인

2

- के

$\therefore \frac{\pi}{5}$

드

邨।

I +2

i

욜

ํ.

a

등

政

ฮี

ธ.

๖잏

ญั้

을

象实

웡

트

E.

중응 늘

플

으

르믐

늘

¿

气 :

⿶ี้

1

- 可帝

을 के

은 픙 


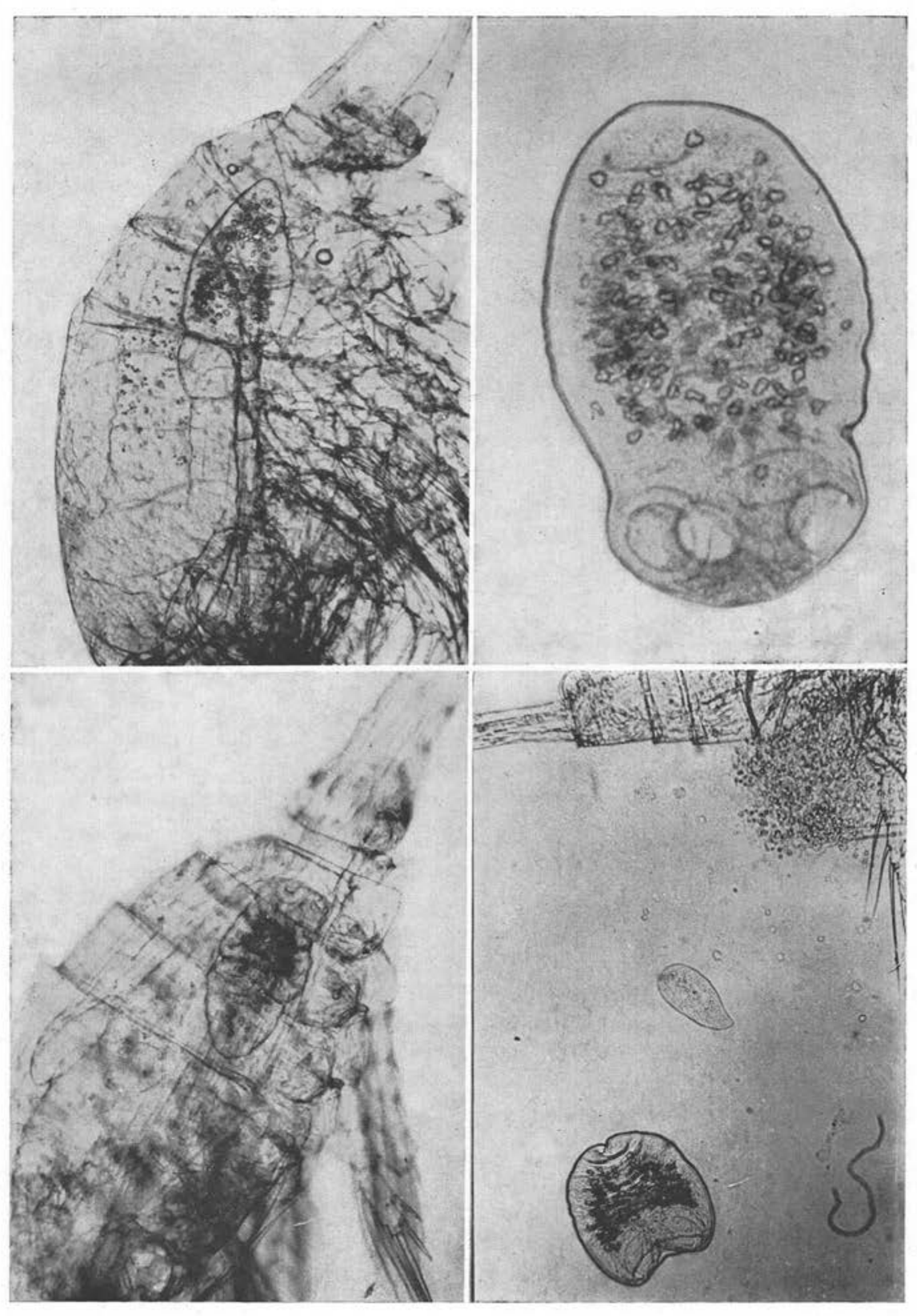

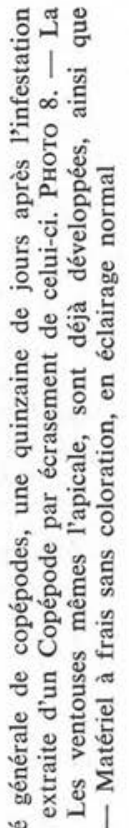

敞

हु एँ

올 正

ฮี ธี

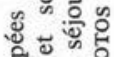

\%ั. 동

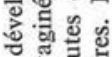

苛. 䓃

है क

氙 总

हั ฏ

ऽ 콜

ฆै। 总 땡

ปึ一

1 。

ง.올 닌

ऽ.

的通

용

웡

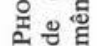


$2^{\circ}$ Des jeunes anguilles de $15 \mathrm{~cm}$ environ de long (élevages en laboratoire de jeunes civelles) subirent également un repas infectant.

- Au cours d'un premier essai, deux jeunes anguilles furent mises en contact avec des Cyclops strenuus infectés depuis 75 jours environ. La dissection de ces anguilles 15 jours après le repas infectant se révéla négative. Cependant, dans ce premier essai, l'ingestion des Copépodes par les anguilles n'avait pas été contrôlée.

- Au cours d'un deuxième essai, quatre jeunes anguilles furent infestées individuellement avec des Cyclops abyssorum infectés depuis 60 jours.

L'ingestion des Copépodes fut ici contrôlée. Les jeunes anguilles furent succes-

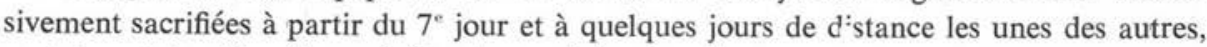
mais leur tube digestif se révéla chaque fois négatif.

$3^{\circ}$ Deux jeunes perches du Canada [Eupomotis gibbosus (L.)] furent également utilisées. Elles reçurent des fragments de vers de terre dans lesquels avaient été inclus plusieurs Cyclops abyssorum infectés depuis 60 jours. Chaque perche reçut ainsi environ 15 Cyclops, soit environ 45 larves de Protéocéphale. Sacrifiées respectivement 24 et 72 heures après le repas infectant, elles révélèrent un tube digestif négatif.

\section{Discussion}

Il ne nous fut malheureusement pas possible de tenter des infestations avec des Poissons appartenant à la même espèce que ceux à l'origine du matériel de départ, c'est-à-dire des Coregonus fera. Il nous est donc impossible de conclure sur les modalités du cycle évolutif complet, notamment sur l'éventualité de l'intervention dans ce cycle d'un deuxième hôte intermédiaire. Cependant, nous pensons qu'il existe une très forte présomption pour qu'il n'y ait pas deux hôtes intermédiaires et que l'infestation de l'hôte définitif, le Coregonus fera, se fasse directement par ingestion de Copépodes infectés. Cette opinion a pour bases, d'une part, la morphologie des larves à maturité observées chez les Copépodes, morphologie relativement proche de celle des Cestodes adultes récoltés chez les Poissons, d'autre part, le régime exclusivement zooplanctonique des Coregonus fera. En effet, de nombreuses dissections de tubes digestifs de Coregonus fera, prélevés là où le plancton ayant servi à nos expérimentations fut récolté, ont montré que la nourriture ingérée par ces Poissons était constituée uniquement de zooplancton, et cela même chez des individus de très grosse taille.

Le problème se pose de savoir à quelle espèce de Protéocéphale appartiennent les adultes que nous avons trouvés chez Coregonus fera et les œufs utilisés pour notre expérimentation. Bien que nous disposions des préparations fixées et colorées de plusieurs Cestodes adultes, la détermination spécifique ne nous semble guère possible. En effet, peu de caractères morphologiques de valeur certaine sont présentés par les diverses espèces de Cestodes du genre Proteocephalus.

De plus, la plupart des descriptions sont fort anciennes et incomplètes, ne donnant pas notamment plusieurs des caractères morphologiques utilisés actuellement pour la diagnose. 
Aussi, en dépit d'une étude morphologique très complète (3), il nous est impossible de mettre un nom en certitude sur notre Cestode. La comparaison avec les diverses espèces décrites antérieurement chez Coregonus fera, ainsi que chez d'autres Salmonidés, soit en provenance d'Europe, notamment des grands lacs suisses et allemands, soit d'Amérique du Nord, ainsi que chez la perche, nous permet d'écarter l'identification à de nombreuses espèces, notamment à Proteocephalus perçae (Müller, 1780), Proteocephalus dubius La Rue 1911 et Proteocephalus exiguus La Rue 1911, d'abord pour des raisons de morphologie, puis pour des raisons de spécificité, du moins en ce qui concerne les deux premières espèces précitées. Notre Protéocéphale se rapproche, par contre, de Proteocephalus neglectus La Rue 1911 et de Proteocephalus pollanicola Gresson et Corbett 1954. Cependant, la description de ces deux espèces présente, avec celle du Protéocéphale que nous avons utilisé pour notre expérimentation, certaines différences morphologiques secondaires sensibles. Il est cependant douteux qu'il s'agisse là d'une nouvelle espèce, car quatre espèces au moins ont déjà été décrites chez le seul Coregonus fera, certaines d'entre elles d'ailleurs ayant été signalées dans le Lac Léman chez ce Poisson.

\section{Bibliographie}

Cooper (A. R.), 1921. - Trematodes and Cestodes of the Canadian artic expedition 1913-18. Rep. of the Canad. arct. exped. 1913-18, 9, part G-H.

Doby (J. M.) et JARecKa (L.), 1964. - Redescription d'un Proteocephalus (Cestode) parasite du poisson Coregonus fera, en provenance du Lac Léman. Problèmes posés par la diagnose spécifique des Cestodes du Genre Proteocephalus. Bull. Soc. Zool. de France, 89, 675.

Gresson (R. A. R.) et Corbett (M. P.), 1954. - A morphological study of a fish tapeworm, Proteocephalus pollanicola. Parasitology, 44, 34.

Joyeux (C.) et BAer (J. G.), 1936. - Cestodes - Faune de France, Paris, Lechevalier édit.

LA RUE (G. R.), 1909. - On the morphology and development of a new Cestode of the genus Proteocephalus Weinland. Trans. of the American microsc. Soc., 29, 17.

LA RUE (G. R.), 1914. - A revision of the cestode family Proteocephalidae, Thèse de Doctorat de l'Univ. d'Hllinois, 1911. In Illinois biological monographs, $1, \mathrm{n}^{\circ \mathrm{s}} 1$ et 2 .

Mac Lulich (D. A.), 1943. - Proteocephalus parallacticus, a new species of tapeworm from lake trout, Cristivomer namaycush. Canad. Jl. of Res., sect. D., 21, 145.

WARdLE (R. A.) et Mc Leod (J. A.), 1952. - The Zoology of tapeworms, North Central Publ. Co., St Paul, Minnesota.

Yamaguti (S.), 1959. - Systema helminthum, vol. 2 (The Cestodes of Vertebrates), NewYork, Intersc. Publ.

Laboratoire de Parasitologie et de Zoologie appliquée de la Faculté de Médecine de Rennes (Professeur J.-M. DoBY)

et Station de recherches hydrobiologiques de I'I.N.R.A., à Thonon

(Directeur : P. LAURENT) 1964).

(3) La description de ce protéocéphale a fait l'objet d'un travail particulier (Doby et Jarecka, 